\title{
Parameter Identifiability of Ship Manoeuvring Modeling Using System Identification
}

\author{
Weilin Luo ${ }^{1,2}$ \\ ${ }^{1}$ School of Mechanical Engineering and Automation, Fuzhou University, Fuzhou 350116, China \\ ${ }^{2}$ Fujian Province Key Laboratory of Structural Performances in Ship and Marine Engineering, Fuzhou 350116, China
}

Correspondence should be addressed to Weilin Luo; wlluofzu@gmail.com

Received 9 June 2016; Revised 17 October 2016; Accepted 19 October 2016

Academic Editor: Laurent Mevel

Copyright (C) 2016 Weilin Luo. This is an open access article distributed under the Creative Commons Attribution License, which permits unrestricted use, distribution, and reproduction in any medium, provided the original work is properly cited.

\begin{abstract}
To improve the feasibility of system identification in the prediction of ship manoeuvrability, several measures are presented to deal with the parameter identifiability in the parametric modeling of ship manoeuvring motion based on system identification. Drift of nonlinear hydrodynamic coefficients is explained from the point of view of regression analysis. To diminish the multicollinearity in a complicated manoeuvring model, difference method and additional signal method are employed to reconstruct the samples. Moreover, the structure of manoeuvring model is simplified based on correlation analysis. Manoeuvring simulation is performed to demonstrate the validity of the measures proposed.
\end{abstract}

\section{Introduction}

Prediction of ship manoeuvrability at the ship design stage is an important method for evaluating the manoeuvring performances of ships [1]. In general, two ways are available to obtain the manoeuvrability parameters (e.g., advance, transfer, tactical diameter, and overshoots), which include simulation-free and simulation-based methods. For the simulation-free method, one can obtain the manoeuvrability parameters directly from database, full-scale trials, or freerunning model tests. For the simulation-based method, either system based manoeuvring simulation or computational fluid dynamics (CFD) based manoeuvring simulation can be employed [2]. No matter which kind of simulation is used, usually the ship manoeuvring model is indispensable, which also determines the accuracy of the prediction of manoeuvrability. Generally, there are two ways to obtain a ship manoeuvring model. The most used way is determination of hydrodynamic derivatives (coefficients) in the mathematical model of ship manoeuvring. The second way is where artificial intelligence techniques are employed to obtain an implicit model (or so-called a black-box model) that describes the input-output mapping characteristics of ship dynamics. Examples are the uses of artificial neural networks (ANN) $[3,4]$ and support vector machines (SVM) $[5,6]$.
To determine the hydrodynamic coefficients in the mathematical model of ship manoeuvring, several methods can be used, including database, empirical formula, captive model test, CFD calculation, and system identification (SI) combined with free-running model test or full-scale trials. Among them, the SI based method provides an effective and practical way to identify the hydrodynamic parameters in the ship manoeuvring models. When combined with free-running model tests, this method requires relatively low cost because it is easy to generate more manoeuvres after a first set of free-running model tests [2]. When combined with full-scale trials, the so-called scale effects due to different Reynolds numbers between that of the ship model and full-scale can be avoided. During last decades, the SI based method has made good progresses and many techniques have been developed in the application to ship manoeuvring modeling. Examples are least squares (LS) regression [7, 8], model reference method (MRM) [9, 10], extend Kalman filter (EKF) [11, 12], maximum likelihood (ML) estimation [13, 14], recursive prediction error (RPE) method [15, 16], frequency spectrum analysis (FSA) method [17, 18], particle swarm optimization (PSO) $[19,20]$, genetic algorithm (GA) [21, 22], and support vector regression (SVR) $[23,24]$. Generally, there have been many SI applications to the parameter identification of ship manoeuvring models. 
Like the other means to come to manoeuvring predictions, SI has its own advantages and disadvantages when applied to ship manoeuvring modeling. The main disadvantage is the problem of parameter identifiability. It can be explained from two aspects. One kind of parameter identifiability is whether the parameters can be identified or not. When solving a state space equation with respect to the ship manoeuvring motion, some parameters cannot be identified for instance the added masses or acceleration derivatives, no matter what input-output samples are provided and no matter which SI technique is adopted. The other kind of parameter identifiability is the effect of parameter drift. The identified coefficients might be not physically correct but be mathematically correct [2], which implies that even if the identified parameters deviate from their true values, the manoeuvring model can predict the manoeuvring motion well, compared with the test results. However, this prediction ability is usually restricted to the prediction of the manoeuvre which provides the data samples for system identification. For example, a manoeuvring model for a ship based on the identification of $10^{\circ} / 10^{\circ}$ zigzag manoeuvre can predict the same manoeuvre well (i.e., $10^{\circ} / 10^{\circ}$ zigzag manoeuvre) but fails when predicting other manoeuvres, for instance, $20^{\circ} / 20^{\circ}$ zigzag manoeuvre or turning circle manoeuvres. The effect of parameter drift happens more frequently and severely for a complicated ship manoeuvring model in which a number of hydrodynamic coefficients are contained, for instance, the Abkowitz model.

SI application to the modeling of ship manoeuvring had ever been widely studied in the 1980s with the development of SI techniques for instance the applications of EKF, ML, and RPE. Correspondingly, the issues on parameter identifiability were addressed and some measures had been proposed [11, $25,26]$. In the 1990s, studies on the application of SI to the ship manoeuvring had been paid relatively less attention to due to a new charming method (well known as the CFD method) and the limitations of test technology and SI techniques available, including the system observability, the sensitivity to the initial estimation of parameters and noises, and the parameter identifiability especially the effect of parameter drift. With the development of modern measurement equipment and the introduction of advanced SI techniques, SI based modeling of ship manoeuvring received renewed attention during the past decade. On one hand, some classical SI techniques have been continuously applied and developed, for instance, the LS method [8], the EKF method [12], the RPE method [16], and some hybrid methods $[27,28]$. On the other hand, several novel and interesting SI methods have been proposed. Representative examples are the artificial intelligence techniques including PSO [19, 20], GA [21, 22], SVM [23, 24], and ANN [29-31]. However, it should be noted that much work (including the work on classical SI or the work on new SI) concerned little about the problem of parameter identifiability especially for the effect of parameter drift or avoided dealing with the problem by selecting a simple manoeuvring model (e.g., the Nomoto model or the response model) in which the parameter drift is weak because few parameters are involved.

In this paper, some issues on the parameter identifiability are summarized. Furthermore, several measures to deal with the identifiability problem are presented especially for the diminishment of the parameter drift in the identification of a complicated ship manoeuvring model, the Abkowitz model. Combined with free-running model test results, SVM based system identification is employed to obtain the hydrodynamic coefficients. Based on the identified manoeuvring model, manoeuvring simulation is conducted and prediction results are compared with the test results, which demonstrates the validity of the proposed methods.

\section{Determination of Added Masses and Added Moment of Inertia}

Three kinds of models are available for the prediction of ship manoeuvring, including the Abkowitz model [32], the MMG model [33], and the response model [34]. Comparatively, the first two models are frequently used because they give a more comprehensive understanding of the ship manoeuvring motion. On the other hand, a number of hydrodynamic coefficients are contained in the models, which makes it challenging to determine these coefficients accurately. From the point of view of system identification, one of the difficulties is that not all coefficients can be identified.

Taking a linear manoeuvring model as an example, the state space equation can be written as

$$
\begin{aligned}
& {\left[\begin{array}{ccc}
m-X_{\dot{u}} & 0 & 0 \\
0 & m-Y_{\dot{v}} & m x_{G}-Y_{\dot{r}} \\
0 & m x_{G}-N_{\dot{v}} & I_{z z}-N_{\dot{r}}
\end{array}\right]\left[\begin{array}{c}
\dot{u} \\
\dot{v} \\
\dot{r}
\end{array}\right]} \\
& \quad=\left[\begin{array}{ccc}
X_{u} & 0 & 0 \\
0 & Y_{v} & Y_{r}-m u_{0} \\
0 & N_{v} & N_{r}-m x_{G} u_{0}
\end{array}\right]\left[\begin{array}{l}
u \\
v \\
r
\end{array}\right]+\left[\begin{array}{c}
0 \\
Y_{\delta} \\
N_{\delta}
\end{array}\right] \delta,
\end{aligned}
$$

where $u$ is the surge speed, $v$ is the sway speed, $r$ is the yaw rate, $\delta$ is the rudder angle, $m$ is the mass of the ship, $I_{z z}$ is the moment of inertia of the ship, $x_{G}$ longitudinal coordinate of the ship's center of gravity, $u_{0}$ is the service speed of the ship, $X_{\dot{u}}$ et al. are the hydrodynamic derivatives among which the acceleration derivatives $-X_{\dot{u}},-Y_{\dot{v}},-Y_{\dot{r}},-N_{\dot{v}}$ are named as the added masses, and $-N_{\dot{r}}$ is the added moment of inertia. Denote

$$
\begin{aligned}
& X=\left[\begin{array}{l}
u \\
v \\
r
\end{array}\right], \\
& P=\left[\begin{array}{ccc}
m-X_{\dot{u}} & 0 & 0 \\
0 & m-Y_{\dot{v}} & m x_{G}-Y_{\dot{r}} \\
0 & m x_{G}-N_{\dot{v}} & I_{z z}-N_{\dot{r}}
\end{array}\right], \\
& A=\left[\begin{array}{ccc}
X_{u} & 0 & 0 \\
0 & Y_{v} & Y_{r}-m u_{0} \\
0 & N_{v} & N_{r}-m x_{G} u_{0}
\end{array}\right], \\
& B=\left[\begin{array}{c}
0 \\
Y_{\delta} \\
N_{\delta}
\end{array}\right] .
\end{aligned}
$$


TABLE 1: Added masses and added moment of inertia $\left(\times 10^{-5}\right)$.

\begin{tabular}{lcc}
\hline Coefficient & Slender-body theory & Captive model test \\
\hline$X_{\dot{u}}^{\prime}$ & -39.9 & -42 \\
$Y_{\dot{v}}^{\prime}$ & -728 & -748 \\
$Y_{\dot{r}}^{\prime}$ & 0 & -9.354 \\
$N_{\dot{v}}^{\prime}$ & 0 & 4.646 \\
$N_{\dot{r}}^{\prime}$ & -60.7 & -43.8 \\
\hline
\end{tabular}

Equation (1) can be written as

$$
\dot{X}=P^{-1} A X+P^{-1} B \delta,
$$

if $P$ is invertible.

Using SI, two matrix combinations in (3), that is, $P^{-1} A$ and $P^{-1} B$, can be determined; however, the three coefficient matrices $P, A$, and $B$ cannot be obtained separately. One solution is to determine one coefficient matrix first in another way, for example, the slender-body theory, empirical formula, captive model test, or CFD calculation. Usually but not always, the mass matrix $P$ is selected to be predetermined in that the abovementioned methods have been successfully applied to determine the added masses and added moment of inertia for example [35-38]. Among these methods, the slender-body theory provides a simple but effective way. Table 1 lists the comparison results of nondimensional added masses and added moment of inertia, between the calculation based on slender-body theory [39] and captive model tests for a Mariner ship [40]. It is assumed that the ship is symmetrical about $y z$ plane in calculation, as such $Y_{\dot{r}}^{\prime}=N_{\dot{v}}^{\prime}=0$.

As can be seen from the comparison results, the slenderbody theory gives a satisfactory estimation of three important hydrodynamic derivatives $X_{\dot{u}}^{\prime}, Y_{\dot{v}}^{\prime}$, and $N_{\dot{r}}^{\prime}$. Combined with free-running model tests, SVM based SI was used to identify the hydrodynamic derivatives in an Abkowitz model, except for the added masses and added moment of inertia that were obtained by slender-body method [24].

\section{Parameter Drift and Diminishment Measures}

Another kind of parameter identifiability, referred to as the parameter drift or simultaneous drift of parameters, is more difficult to deal with, especially for the identification of a complicated manoeuvring model such as an Abkowitz model or a nonlinear MMG model. As aforementioned, when parameter drift happens, the identified parameters might be mathematically correct but physically incorrect. Even worse, the "mathematically correct" parameters cannot be obtained.

The reason for the drift of hydrodynamic coefficients has not been made fully clear physically. Hwang applied the slender-body theory to explain the mechanism of the drift of linear hydrodynamic coefficients which is due to the dynamic cancellation [25]. However, for the drift of nonlinear hydrodynamic coefficients, it can only be explained from the point of view of regression analysis, not physically but statistically. The drift of nonlinear hydrodynamic coefficients is due to the so-called multicollinearity [23]. In fact, many nonlinear hydrodynamic coefficients in the mathematical model of ship manoeuvring motion have no clear physical meaning.

3.1. Simultaneous Drift of Linear Hydrodynamic Coefficients. Brief explanation of the simultaneous drift of linear hydrodynamic coefficients is given as follows. More details can be found in $[11,25]$.

For a slender ship moving on the horizontal plane, the viscous sway force and yaw moment can be calculated as [39]

$$
\begin{aligned}
& Y=-\frac{1}{2} \rho \pi d^{2} u_{0}\left(v+\frac{1}{2} L r\right), \\
& N=-\frac{1}{4} \rho \pi d^{2} L u_{0}\left(v+\frac{1}{2} L r\right),
\end{aligned}
$$

where $\rho$ is the fluid density, $d$ is the draft of the ship, $u_{0}$ is the service speed of the ship assuming $u \approx u_{0}$, and $L$ the length of ship. The above two expressions can be rewritten as

$$
\begin{aligned}
& Y=Y_{v} v+Y_{r} r, \\
& N=N_{v} v+N_{r} r,
\end{aligned}
$$

in terms of hydrodynamic derivatives and motion variables. It can be inferred that

$$
\frac{Y_{v}}{Y_{r}}=\frac{N_{v}}{N_{r}}=\frac{2}{L} .
$$

During a ship manoeuvre, the pivot of the ship is near to the bow; therefore, it follows that

$$
v+\frac{1}{2} L r \approx 0
$$

which indicates that

$$
\begin{gathered}
Y_{v} v+Y_{r} r \approx 0, \\
N_{v} v+N_{r} r \approx 0 .
\end{gathered}
$$

Combined with (6), it can be inferred that two pairs of parameters, that is, $Y_{v}$ and $Y_{r}, N_{v}$, and $N_{r}$, are allowed to become larger or smaller simultaneously. Figure 1 presents the simulation results of nondimensional linear components of sway force and yaw moment for the $10^{\circ} / 10^{\circ}$ zigzag manoeuvre of a Mariner vessel [40]. As can be seen, the sway force and yaw moment are small at the helming stage when rudder angle switches. At the steady stage when rudder angle keeps unchanged, the following approximations hold:

$$
\begin{aligned}
Y_{v}^{\prime} v^{\prime}+Y_{r}^{\prime} r^{\prime} & \approx k_{1} Y_{\delta}^{\prime} \delta^{\prime}, \\
N_{v}^{\prime} v^{\prime}+N_{r}^{\prime} r^{\prime} & \approx k_{2} N_{\delta}^{\prime} \delta^{\prime}, \\
v^{\prime} & \approx k_{3} \delta^{\prime}, \\
r^{\prime} & \approx k_{4} \delta^{\prime},
\end{aligned}
$$

where $k_{1 \sim 4}$ are constants. Based on the above approximations, two linear expressions can be obtained as

$$
\begin{gathered}
k_{3} Y_{v}^{\prime}+k_{4} Y_{r}^{\prime} \approx k_{1} Y_{\delta}^{\prime} \\
k_{3} N_{v}^{\prime}+k_{4} N_{r}^{\prime} \approx k_{2} N_{\delta}^{\prime}
\end{gathered}
$$



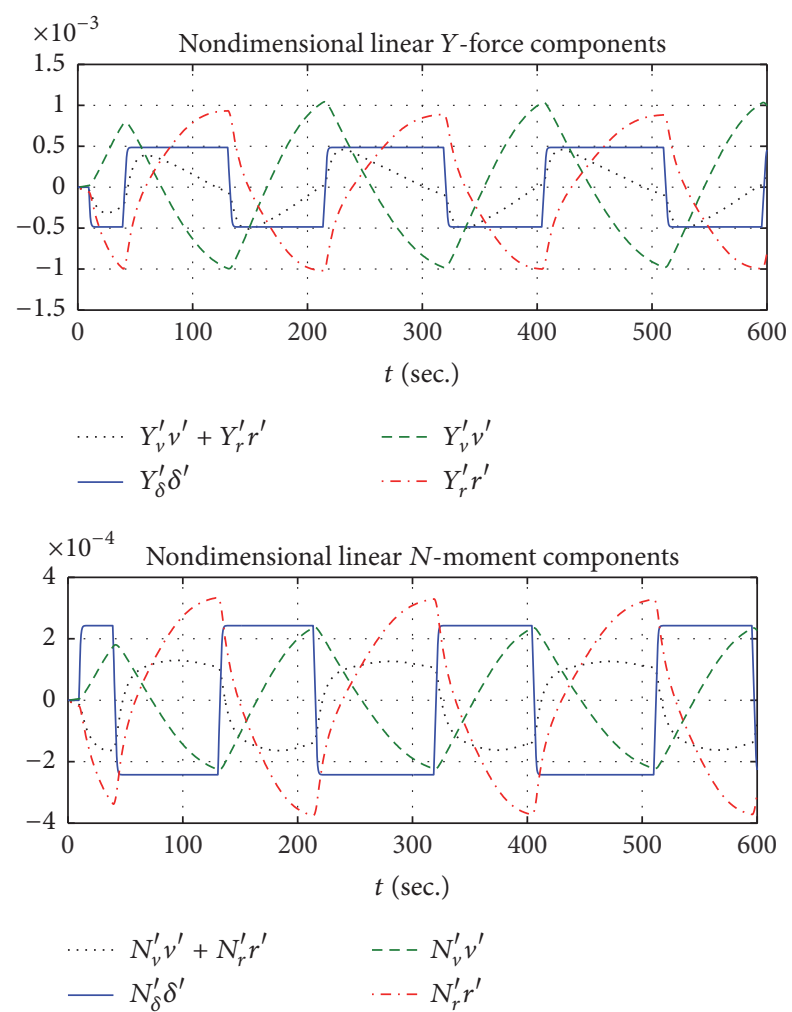

Figure 1: Nondimensional linear components of sway force and yaw moment for $10^{\circ} / 10^{\circ}$ zigzag manoeuvre of a Mariner vessel.

which means that the elements within the parameter set $\left\{Y_{v}^{\prime}, Y_{r}^{\prime}, Y_{\delta}^{\prime}\right\}$ or $\left\{N_{v}^{\prime}, N_{r}^{\prime}, N_{\delta}^{\prime}\right\}$ possibly drift simultaneously.

3.2. Drift of Nonlinear Hydrodynamic Coefficients. From the point of view of regression analysis, the drift of hydrodynamic coefficients, linear and/or nonlinear, results from the socalled multicollinearity. It can be explained that if the input variables of a mathematical model are strongly linearly dependent on each other, their corresponding coefficients in the regression model might be incorrect, even if the predicted results based on the regression model do agree well with the desired. The case is more possible and serious especially when the system contains a large number of parameters to be identified. Equations (8) and (9) present simple examples of linear dependence. It can be inferred that $v$ and $r$ are linearly dependent on each other. This implies that there exists multicollinearity in the model, which results in the parameter drift of $Y_{v}$ and $Y_{r}$ for (8) and $N_{v}$ and $N_{r}$ for (9).

It is noted that multicollinearity is a commonplace in the regression analysis [41], not a special effect that happens in the system identification of ship manoeuvring. Moreover, this phenomenon cannot be eliminated but only moderated or diminished, because the input variables are always linearly dependent on each other, more or less.

It is important to assess the degree of multicollinearity in a system before diminishment measures are taken. Common used assessment tools include $F$ - and $t$-tests, Variance Inflation Factor (VIF) or tolerance (reciprocal of VIF), eigenanalysis, determination coefficient, and correlation coefficient. The last method provides a simple and effective way. Using this method, a correlation coefficient between two variables $x_{1}$ and $x_{2}$ can be defined as

$$
\rho_{x_{1} x_{2}}=\frac{\operatorname{cov}\left(x_{1}, x_{2}\right)}{\sqrt{D\left(x_{1}\right) D\left(x_{2}\right)}},
$$

where $D\left(x_{1}\right)$ and $D\left(x_{2}\right)$ denote the variance and $\operatorname{cov}\left(x_{1}, x_{2}\right)$ denotes the covariance. Usually, the nearer to one the absolute value of $\rho_{x_{1} x_{2}}$ approximates, the more serious the linear dependence between $x_{1}$ and $x_{2}$ is. An example is given with respect to a nonlinear ship manoeuvring model, in which the nondimensional yaw-equation can be described as

$$
\begin{aligned}
& \left(m^{\prime} x_{G}^{\prime}-N_{\dot{v}}^{\prime}\right) \Delta \dot{v}^{\prime}+\left(I_{z z}^{\prime}-N_{\dot{r}}^{\prime}\right) \Delta \dot{r}^{\prime} \\
& =\Delta f_{3}^{\prime}\left(\Delta u^{\prime}, \Delta v^{\prime}, \Delta r^{\prime}, \Delta \delta^{\prime}\right),
\end{aligned}
$$

where $\Delta u^{\prime}, \Delta v^{\prime}, \Delta r^{\prime}$, and $\Delta \delta^{\prime}$ are small perturbations from nominal values of surge speed, sway speed, yaw rate, and rudder angle, respectively. The nonlinear function $\Delta f_{3}^{\prime}$ on the right-hand side of (13) can be expressed as [42]

$$
\begin{aligned}
\Delta f_{3}^{\prime}= & N_{v}^{\prime} \Delta v^{\prime}+N_{r}^{\prime} \Delta r^{\prime}+N_{\delta}^{\prime} \Delta \delta^{\prime}+N_{|v| v}^{\prime}\left|\Delta v^{\prime}\right| \Delta v^{\prime} \\
& +N_{|v| r}^{\prime}\left|\Delta v^{\prime}\right| \Delta r^{\prime}+N_{|r| r}^{\prime}\left|\Delta r^{\prime}\right| \Delta r^{\prime} \\
& +N_{v|r|}^{\prime} \Delta v^{\prime}\left|\Delta r^{\prime}\right|+N_{\delta \delta \delta}^{\prime} \Delta \delta^{\prime 3}+N_{v v \delta}^{\prime} \Delta v^{\prime 2} \Delta \delta^{\prime} \\
& +N_{v v \delta}^{\prime} \Delta v^{\prime} \Delta \delta^{\prime 2}+N_{r \delta \delta}^{\prime} \Delta r^{\prime} \Delta \delta^{\prime 2}+N_{r r \delta}^{\prime} \Delta r^{\prime 2} \Delta \delta^{\prime} \\
& +N_{r v \delta}^{\prime} \Delta r^{\prime} \Delta v^{\prime} \Delta \delta^{\prime}+N_{0}^{\prime} .
\end{aligned}
$$

Combined with the test data of $25^{\circ} / 5^{\circ}$ zigzag manoeuvre of a KVLCC2 ship which was conducted in the Hamburg Ship Model Basin (HSVA), the correlation coefficients of variables in (14) can be calculated, as listed in Table 2. As can be seen, the degree of linear dependence is heavy for some variables for instance $\Delta v^{\prime}$ and $\Delta v^{\prime} \Delta \delta^{\prime 2}$, with the correlation coefficient 0.99 . A heavy multicollinearity might exist with respect to the system (14) which results in the parameter drift, not only for the linear parameters but also the nonlinear parameters.

3.3. Measures to Diminish the Drift of Hydrodynamic Coefficients. From the above discussion, it can be concluded that multicollinearity is the main reason for the drift of hydrodynamic coefficients especially for the nonlinear ones. Therefore, the alleviation of multicollinearity is vital for the diminishment of parameter drift.

Several remedies are available to moderate the degree of multicollinearity for instance difference method, pruningvariable method [43], principal component regression (PCR) analysis [44], partial least squares (PLS) [45], and a group method of data handling (GMDH) [46]. Some of them were developed to diminish the drift of hydrodynamic coefficients. For example, Hwang proposed parallel processing, exaggerated over- and underestimation, parameter transformation to moderate the drift of linear hydrodynamic coefficients [25]. 
TABLE 2: Matrix of correlation coefficients.

\begin{tabular}{|c|c|c|c|c|c|c|c|c|c|c|c|c|c|}
\hline & $N_{v}^{\prime}$ & $N_{r}^{\prime}$ & $N_{|v| v}^{\prime}$ & $N_{|v| r}^{\prime}$ & $N_{\delta}^{\prime}$ & $N_{\delta \delta \delta}^{\prime}$ & $N_{v \delta \delta}^{\prime}$ & $N_{v v \delta}^{\prime}$ & $N_{|r| r}^{\prime}$ & $N_{v|r|}^{\prime}$ & $N_{r \delta \delta}^{\prime}$ & $N_{r r \delta}^{\prime}$ & $N_{r v \delta}^{\prime}$ \\
\hline$N_{v}^{\prime}$ & 1.00 & 0.45 & 0.98 & 0.67 & 0.91 & 0.92 & 0.99 & 0.97 & 0.39 & 0.84 & 0.54 & 0.54 & 0.68 \\
\hline$N_{r}^{\prime}$ & & 1.00 & 0.42 & 0.84 & 0.53 & 0.54 & 0.47 & 0.43 & 0.97 & 0.66 & 0.91 & 0.75 & 0.81 \\
\hline$N_{|v| v}^{\prime}$ & & & 1.00 & 0.66 & 0.83 & 0.85 & 0.98 & 1.00 & 0.32 & 0.77 & 0.50 & 0.42 & 0.67 \\
\hline$N_{|v| r}^{\prime}$ & & & & 1.00 & 0.62 & 0.64 & 0.68 & 0.66 & 0.75 & 0.87 & 0.92 & 0.73 & 1.00 \\
\hline$N_{\delta}^{\prime}$ & & & & & 1.00 & 0.99 & 0.91 & 0.83 & 0.50 & 0.83 & 0.61 & 0.71 & 0.62 \\
\hline$N_{\delta \delta \delta}^{\prime}$ & & & & & & 1.00 & 0.92 & 0.85 & 0.50 & 0.83 & 0.62 & 0.69 & 0.64 \\
\hline$N_{v \delta \delta}^{\prime}$ & & & & & & & 1.00 & 0.98 & 0.40 & 0.83 & 0.55 & 0.53 & 0.69 \\
\hline$N_{v v \delta}^{\prime}$ & & & & & & & & 1.00 & 0.33 & 0.77 & 0.50 & 0.42 & 0.67 \\
\hline$N_{|r| r}^{\prime}$ & & & & & & & & & 1.00 & 0.62 & 0.86 & 0.81 & 0.72 \\
\hline$N_{v|r|}^{\prime}$ & & & & & & & & & & 1.00 & 0.78 & 0.82 & 0.89 \\
\hline$N_{r \delta \delta}^{\prime}$ & & & & & & & & & & & 1.00 & 0.83 & 0.91 \\
\hline$N_{r r \delta}^{\prime}$ & & & & & & & & & & & & 1.00 & 0.73 \\
\hline$N_{r v \delta}^{\prime}$ & & & & & & & & & & & & & 1.00 \\
\hline
\end{tabular}

For nonlinear hydrodynamic coefficients, ridge regression and modification of input scenario were proposed [47, 48]. However, for given samples from real manoeuvring tests, such a modification of excitation might be not applicable; in other words, this approach might only work for simulation data.

Since the multicollinearity is an inherent characteristic of a regression model, besides the regression tool (e.g., LS and EKF), modification of the model is a direct and effective way to alleviate the multicollinearity, which can be achieved by altering the structure of the model and reconstructing the input variables in the model, with the aim to decrease the linear dependence of variables. Based on these ideas, three approaches are presented to diminish the drift of hydrodynamic coefficients, including simplification of the manoeuvring model, difference of the samples, and the method of additional signal. Studies are performed mainly combined with the Abkowitz model.

3.3.1. Simplification of the Manoeuvring Model. Although the Abkowitz model is often preferred in the prediction of ship manoeuvring, such a "large" parametric system makes it inconvenient to use in practice. Actually some nonlinear derivatives are difficult to be determined although they could be unimportant. In practical application of the Abkowitz model, simplification had been performed to improve the feasibility of this model. Examples are the work by Abkowitz [11] and Norrbin Nils [37]. Physical meanings were the main concern in the simplification. The simplification can also be performed from the point of view of regression analysis [24]. Taking the yaw-equation (13) as an example, the original expression of (14) is

$$
\begin{aligned}
\Delta f_{3}^{\prime}= & N_{0 u}^{\prime} \Delta u^{\prime}+N_{0 u u}^{\prime} \Delta u^{\prime 2}+N_{v}^{\prime} \Delta v^{\prime}+N_{r}^{\prime} \Delta r^{\prime} \\
& +N_{\delta}^{\prime} \Delta \delta^{\prime}+N_{v v v}^{\prime} \Delta v^{\prime 3}+N_{\delta \delta \delta}^{\prime} \Delta \delta^{\prime 3} \\
& +N_{v v r}^{\prime} \Delta v^{\prime 2} \Delta r^{\prime}+N_{v v \delta}^{\prime} \Delta v^{\prime 2} \Delta \delta^{\prime}+N_{v \delta \delta}^{\prime} \Delta v^{\prime} \Delta \delta^{\prime 2}
\end{aligned}
$$

$$
\begin{aligned}
& +N_{\delta u}^{\prime} \Delta \delta^{\prime} \Delta u^{\prime}+N_{v u}^{\prime} \Delta v^{\prime} \Delta u^{\prime}+N_{r u}^{\prime} \Delta r^{\prime} \Delta u^{\prime} \\
& +N_{\delta u u}^{\prime} \Delta \delta^{\prime} \Delta u^{\prime 2}+N_{r r r}^{\prime} \Delta r^{\prime 3}+N_{v r r}^{\prime} \Delta v^{\prime} \Delta r^{\prime 2} \\
& +N_{v u u}^{\prime} \Delta v^{\prime} \Delta u^{\prime 2}+N_{r u u}^{\prime} \Delta r^{\prime} \Delta u^{\prime 2}+N_{r \delta \delta}^{\prime} \Delta r^{\prime} \Delta \delta^{\prime 2} \\
& +N_{r r \delta}^{\prime} \Delta r^{\prime 2} \Delta \delta^{\prime}+N_{r v \delta}^{\prime} \Delta r^{\prime} \Delta v^{\prime} \Delta \delta^{\prime}+N_{0}^{\prime},
\end{aligned}
$$

in which 22 hydrodynamic coefficients are contained [40]. To simplify it, all the nonlinear terms related to the surge perturbation $\Delta u^{\prime}$ are eliminated for two reasons. First, since the ship total velocity $U(t)$ is taken as the nondimensional factor, the effect of velocity loss has been taken into account in every velocity term. Second, the linear dependence between the linear and nonlinear terms relating to $\Delta u^{\prime}$ is significant, which will result in the parameter drift. For example, the third and twelfth terms on the right hand side of (15) at the $k$ th sampling time can be written as

$$
N_{v}^{\prime} \Delta v(k) U(k)+N_{v u}^{\prime} \Delta v(k) \Delta u(k)
$$

by considering the prime nondimensional definition

$$
\begin{aligned}
\Delta u^{\prime} & =\frac{\Delta u}{U}, \\
\Delta \dot{u}^{\prime} & =\frac{\Delta \dot{u}}{\left(U^{2} / L\right)} .
\end{aligned}
$$

For moderate manoeuvres, it holds that

$$
U(k) \approx u_{0}+\Delta u(k) .
$$

The expression (16) can be written as

$$
N_{v}^{\prime} \Delta v(k) u_{0}+N_{v}^{\prime} \Delta v(k) \Delta u(k)+N_{v u}^{\prime} \Delta v(k) \Delta u(k) .
$$

Obviously, the second term is linearly dependent on the third term. In consideration of the significance of the linear derivative $N_{v}^{\prime}$, the nonlinear term $N_{v u}^{\prime} \Delta v(k) \Delta u(k)$ is removed. 
In the same way, the other six terms related to the derivatives $N_{0 u \mathcal{u}}^{\prime}, N_{\delta u}^{\prime}, N_{r u}^{\prime}, N_{\delta u \mathcal{u}}^{\prime}, N_{v u u}^{\prime}$, and $N_{r u u}^{\prime}$ can be removed from (15). Ignoring the effect of surge speed $u$ on the change of yaw moment, $N_{0 u}^{\prime} \Delta u^{\prime}$ can be removed furthermore. Finally, by introducing the cross flow models to replace the third-order nonlinear terms, model (15) is simplified to model (14), with the number of hydrodynamic coefficients decreasing from 22 to 14 .

Sensitivity analysis provides another way to simplify the mathematical model of ship manoeuvring [49-53]. Using this method, the effect of the hydrodynamic coefficient on the prediction of ship manoeuvring is studied by observing the change of manoeuvring (e.g., velocity, angular velocity, advance, transfer, overshoots, and tactical diameter) to the change of hydrodynamic coefficients. Based on the results of sensitivity analysis, some trivial hydrodynamic coefficients are removed from the mathematical model. The main difficulties with this method are the determination of two factors: (i) the criteria of importance for a hydrodynamic coefficient which decides this coefficient will be retained or removed from the model; (ii) the reference value of a hydrodynamic coefficient when the real experimental data are used for identification, instead of the simulation data.

3.3.2. Difference Method. Besides simplification of the mathematical model of ship manoeuvring, reconstruction of the input variables or samples provides another effective way to alleviate the parameter drift. It is noted that such a reconstruction does not require a new manoeuvring to obtain new input-output samples for system identification but modify the obtained samples. Difference method provides a simple way to reconstruct the samples [24].

Taking (13) as an example, the following discrete equation can be obtained:

$$
\begin{aligned}
\Delta r(k+1)= & \Delta r(k)+\theta_{1} \Delta v(k) U(k)+\theta_{2} \Delta r(k) U(k) \\
& +\theta_{3} \Delta \delta(k) U^{2}(k)+\theta_{4}|\Delta v(k)| \Delta v(k) \\
& +\theta_{5}|\Delta v(k)| \Delta r(k)+\theta_{6}|\Delta r(k)| \Delta r(k) \\
& +\theta_{7} \Delta v(k)|\Delta r(k)|+\theta_{8} \Delta \delta^{3}(k) U^{2}(k) \\
& +\theta_{9} \Delta v^{2}(k) \Delta \delta(k) \\
& +\theta_{10} \Delta v(k) \Delta \delta^{2}(k) U(k) \\
& +\theta_{11} \Delta r(k) \Delta \delta^{2}(k) U(k) \\
& +\theta_{12} \Delta r^{2}(k) \Delta \delta(k) \\
& +\theta_{13} \Delta r(k) \Delta v(k) \Delta \delta(k)+\theta_{14} U^{2}(k),
\end{aligned}
$$

where $\theta_{1 \sim 14}$ are the linear combinations of the hydrodynamic coefficients with respect to the sway and yaw motion [23]. Define vectors

$$
\begin{aligned}
& \boldsymbol{\Theta}=\left[\theta_{1}, \theta_{2}, \ldots, \theta_{14}\right]_{14 \times 1}, \\
& \mathbf{X}(k)=[\Delta v(k) U(k), \Delta r(k) U(k), \Delta \delta(k) \\
& \quad \cdot U^{2}(k),|\Delta v(k)| \Delta v(k),|\Delta v(k)| \Delta r(k),|\Delta r(k)|
\end{aligned}
$$

$$
\begin{aligned}
& \cdot \Delta r(k), \Delta v(k)|\Delta r(k)|, \Delta \delta^{3}(k) U^{2}(k), \Delta v^{2}(k) \\
& \cdot \Delta \delta(k), \Delta v(k) \Delta \delta^{2}(k) U(k), \Delta r(k) \Delta \delta^{2}(k) \\
& \cdot U(k), \Delta r^{2}(k) \Delta \delta(k), \Delta r(k) \Delta v(k) \\
& \left.\cdot \Delta \delta(k), U^{2}(k)\right]_{14 \times 1}^{T} .
\end{aligned}
$$

Equation (20) can be rewritten as

$$
\Delta r(k+1)=\Delta r(k)+\boldsymbol{\Theta X}(k) .
$$

At a neighbour sampling time, the above equation becomes

$$
\Delta r(k)=\Delta r(k-1)+\boldsymbol{\Theta X}(k-1) .
$$

Combined with (22), the following equality holds:

$$
z(k)=z(k-1)+\Theta \mathbf{Y}(k-1),
$$

by definition of

$$
\begin{aligned}
z(k) & =\Delta r(k+1)-\Delta r(k), \\
\mathbf{Y}(k-1) & =\mathbf{X}(k)-\mathbf{X}(k-1) .
\end{aligned}
$$

Comparing (23) with (24), it can be seen that although the input-output pair are altered, the coefficient matrix remains the same. Usually, if the degree of linear dependence of input variables in the original system is serious, it could be moderated after difference of samples. As a comparison, Table 3 lists the correlation coefficients after the difference method is employed, as shown below the principal diagonal (elements above the principal diagonal are the correlation coefficients without difference method shown in Table 2). As can be seen, the linear dependence of the input variables has been obviously decreased.

3.3.3. Additional Signal. Besides the difference method, another approach to reconstruct the input-output samples can be used, which refers to the method of additional signal. Such a method had been successfully applied to the identification of ship manoeuvring by analyzing simulation data [23] and captive model test results [54]. Taking the system (22) as an example, an additional ramp signal can be designed as

$$
y_{a}(k+1)=y_{a}(k)+\lambda,
$$

where $\lambda$ is a constant. Adding this signal to (22), a new parameter system can be obtained as

$$
\begin{aligned}
& {\left[\Delta r(k+1)+y_{a}(k+1)\right]} \\
& \quad=\left[\Delta r(k)+y_{\mathrm{a}}(k)\right]+\boldsymbol{\Theta} \mathbf{X}(k)+\lambda,
\end{aligned}
$$

where the output variable and the first input variable change; however, the parameter matrix $\Theta$ related to hydrodynamic coefficients does not. The additional signal can be viewed as an additional excitation to the original system. As a result, the manoeuvring characteristics of the original system, which 
TABLE 3: Comparison of correlation coefficients using difference method.

\begin{tabular}{lccccccccccccc}
\hline & $N_{v}^{\prime}$ & $N_{r}^{\prime}$ & $N_{|v| v}^{\prime}$ & $N_{|v| r}^{\prime}$ & $N_{\delta}^{\prime}$ & $N_{\delta \delta \delta}^{\prime}$ & $N_{v \delta \delta}^{\prime}$ & $N_{v v \delta}^{\prime}$ & $N_{|r| r}^{\prime}$ & $N_{v|r|}^{\prime}$ & $N_{r \delta \delta}^{\prime}$ & $N_{r r \delta}^{\prime}$ & $N_{r v \delta}^{\prime}$ \\
\hline$N_{v}^{\prime}$ & $\mathbf{1 . 0 0}$ & 0.45 & 0.98 & 0.67 & 0.91 & 0.92 & 0.99 & 0.97 & 0.39 & 0.84 & 0.54 & 0.54 & 0.68 \\
$N_{r}^{\prime}$ & 0.44 & $\mathbf{1 . 0 0}$ & 0.42 & 0.84 & 0.53 & 0.54 & 0.47 & 0.43 & 0.97 & 0.66 & 0.91 & 0.75 & 0.81 \\
$N_{|v| v}^{\prime}$ & 0.83 & 0.31 & $\mathbf{1 . 0 0}$ & 0.66 & 0.83 & 0.85 & 0.98 & 1.00 & 0.32 & 0.77 & 0.50 & 0.42 & 0.67 \\
$N_{|v| r}^{\prime}$ & 0.34 & 0.21 & 0.38 & $\mathbf{1 . 0 0}$ & 0.62 & 0.64 & 0.68 & 0.66 & 0.75 & 0.87 & 0.92 & 0.73 & 1.00 \\
$N_{\delta}^{\prime}$ & 0.64 & 0.44 & 0.23 & 0.01 & $\mathbf{1 . 0 0}$ & 0.99 & 0.91 & 0.83 & 0.50 & 0.83 & 0.61 & 0.71 & 0.62 \\
$N_{\delta \delta \delta}^{\prime}$ & 0.50 & 0.33 & 0.25 & 0.13 & 0.74 & $\mathbf{1 . 0 0}$ & 0.92 & 0.85 & 0.50 & 0.83 & 0.62 & 0.69 & 0.64 \\
$N_{v \delta \delta}^{\prime}$ & 0.84 & 0.41 & 0.77 & 0.36 & 0.48 & 0.66 & $\mathbf{1 . 0 0}$ & 0.98 & 0.40 & 0.83 & 0.55 & 0.53 & 0.69 \\
$N_{v v \delta}^{\prime}$ & 0.81 & 0.31 & 0.99 & 0.39 & 0.22 & 0.29 & 0.82 & $\mathbf{1 . 0 0}$ & 0.33 & 0.77 & 0.50 & 0.42 & 0.67 \\
$N_{|r| r}^{\prime}$ & 0.34 & 0.88 & 0.03 & 0.07 & 0.54 & 0.35 & 0.28 & 0.04 & $\mathbf{1 . 0 0}$ & 0.62 & 0.86 & 0.81 & 0.72 \\
$N_{v|r|}^{\prime}$ & 0.57 & 0.27 & 0.21 & 0.46 & 0.58 & 0.41 & 0.40 & 0.19 & 0.30 & $\mathbf{1 . 0 0}$ & 0.78 & 0.82 & 0.89 \\
$N_{r \delta \delta}^{\prime}$ & 0.19 & 0.17 & 0.08 & 0.58 & 0.21 & 0.29 & 0.09 & 0.06 & 0.05 & 0.30 & $\mathbf{1 . 0 0}$ & 0.83 & 0.91 \\
$N_{r r \delta}^{\prime}$ & 0.29 & 0.32 & 0.15 & 0.16 & 0.72 & 0.44 & 0.09 & 0.18 & 0.49 & 0.62 & 0.50 & $\mathbf{1 . 0 0}$ & 0.73 \\
$N_{r v \delta}^{\prime}$ & 0.32 & 0.11 & 0.33 & 0.90 & 0.01 & 0.09 & 0.26 & 0.32 & 0.16 & 0.49 & 0.73 & 0.24 & $\mathbf{1 . 0 0}$ \\
\hline & & & & & & & & & & &
\end{tabular}

TABLE 4: Comparison of correlation coefficients using additional signal.

\begin{tabular}{lcc}
\hline & $N_{1}$ & $N_{1}^{\prime}$ \\
\hline$N_{v}^{\prime}$ & 0.465 & 0.081 \\
$N_{r}^{\prime}$ & 0.996 & 0.011 \\
$N_{|v| v}^{\prime}$ & 0.450 & 0.026 \\
$N_{|v| r}^{\prime}$ & 0.849 & 0.002 \\
$N_{\delta}^{\prime}$ & 0.552 & 0.028 \\
$N_{\delta \delta \delta}^{\prime}$ & 0.556 & 0.022 \\
$N_{v \delta \delta}^{\prime}$ & 0.491 & 0.005 \\
$N_{v v \delta}^{\prime}$ & 0.455 & 0.029 \\
$N_{|r| r}^{\prime}$ & 0.023 & 0.834 \\
$N_{v|r|}^{\prime}$ & 0.970 & 0.039 \\
$N_{r \delta \delta}^{\prime}$ & 0.665 & 0.015 \\
$N_{r r \delta}^{\prime}$ & 0.912 & 0.013 \\
$N_{r v \delta}^{\prime}$ & 0.771 & 0.024 \\
\hline
\end{tabular}

might cause the parameter drift as described in the Section 3.1, are changed and the parameter drift can be alleviated resultantly. From the point of view of regression analysis, the introduction of the additional signal alters the input variables, which might alleviate the degree of linear dependence between the changed variables and others. Taking model (27) as an example, assuming $\lambda=1$, Table 4 lists the correlation coefficients before and after the additional signal is attached to the samples, in which $N_{1}=N_{1}^{\prime}=1$ indicates that coefficient of the first input variable $\left[\Delta r(k)+y_{a}(k)\right]$ in (27) is one. As can be seen from the comparison results, the degree of linear dependence remarkably decreases for most of variables after an additional signal is added.

It is noted that the design of additional signal depends on the characteristic of the data samples or the manoeuvring motion. For periodically nonlinear motion such as zigzag manoeuvre or turning circle manoeuvring, the linear ramp signal like (26) works well [23]; for a linear motion such as oblique towing test, a nonlinear additional signal is more appropriate [54].
TABLE 5: Hydrodynamic coefficients of KVLCC2 $\left(\times 10^{-5}\right)$.

\begin{tabular}{cccccccc}
\hline & Value & $X$-coef. & Value & $Y$-coef. & Value & $N$-coef. & Value \\
\hline$X_{\dot{u}}^{\prime}$ & -95.4 & $X_{u}^{\prime}$ & -93 & $Y_{v}^{\prime}$ & -358 & $N_{v}^{\prime}$ & -6.3 \\
$Y_{\dot{v}}^{\prime}$ & -1283 & $X_{v v}^{\prime}$ & 149 & $Y_{r}^{\prime}$ & 1980 & $N_{r}^{\prime}$ & -74 \\
$Y_{\dot{r}}^{\prime}$ & 0 & $X_{r r}^{\prime}$ & -108 & $Y_{\delta}^{\prime}$ & 448 & $N_{\delta}^{\prime}$ & -165 \\
$N_{\dot{v}}^{\prime}$ & 0 & $X_{\delta \delta}^{\prime}$ & -105 & $Y_{|v| v}^{\prime}$ & -3.5 & $N_{|v| v}^{\prime}$ & 20 \\
$N_{\dot{r}}^{\prime}$ & -107 & $X_{v r}^{\prime}$ & -237 & $Y_{|v| r}^{\prime}$ & 30 & $N_{|v| r}^{\prime}$ & -9.9 \\
$x_{G}^{\prime}$ & 3486 & $X_{v \delta}^{\prime}$ & 172 & $Y_{|r| r}^{\prime}$ & 679 & $N_{|r| r}^{\prime}$ & -17 \\
$m^{\prime}$ & 1908 & $X_{r \delta}^{\prime}$ & -433 & $Y_{v|r|}^{\prime}$ & 158 & $N_{v|r|}^{\prime}$ & -43 \\
$I_{z}^{\prime}$ & 119 & $X_{0}^{\prime}$ & -48 & $Y_{\delta \delta \delta}^{\prime}$ & -94 & $N_{\delta \delta \delta}^{\prime}$ & 22 \\
& & & & $Y_{v \delta \delta}^{\prime}$ & -3.6 & $N_{v \delta \delta}^{\prime}$ & 17 \\
& & & & $Y_{v v \delta}^{\prime}$ & -7.3 & $N_{v v \delta}^{\prime}$ & 12 \\
& & & & $Y_{r \delta \delta}^{\prime}$ & -23 & $N_{r \delta \delta}^{\prime}$ & 22 \\
& & & & $Y_{r r \delta}^{\prime}$ & 134 & $N_{r r \delta}^{\prime}$ & -40 \\
& & & & $Y_{r v \delta}^{\prime}$ & -28 & $N_{r v \delta}^{\prime}$ & 3.9 \\
& & & & $Y_{0}^{\prime}$ & -6.6 & $N_{0}^{\prime}$ & 0.2 \\
\hline
\end{tabular}

\section{Example Study}

The abovementioned measures on how to diminish the drift of hydrodynamic coefficients, including difference method and additional signal, are employed in identifying a simplified Abkowitz model combined with free-running model tests of a KVLCC2. SVM based SI is used to obtain the hydrodynamic coefficients. The details of the mathematical model of ship manoeuvring, the SVM methodology, the ship, and the model tests can be found in [24] and relevant literature. Table 5 lists the identification results of the hydrodynamic coefficients, in which the added masses and added moment of inertia are calculated by slender-body theory, as stated in Section 2 . Based on the regression model, $25^{\circ} / 5^{\circ}$ zigzag manoeuvring motion is simulated and the predicted results are compared with the experimental results, as shown in Figure 2. Comparisons with the experimental results of other two manoeuvres are presented in Figure $3\left(20^{\circ} / 5^{\circ}\right.$ zigzag manoeuvre) and Figure $4\left(20^{\circ} / 10^{\circ}\right.$ zigzag manoeuvre), respectively. As can be seen, good agreement between prediction and 


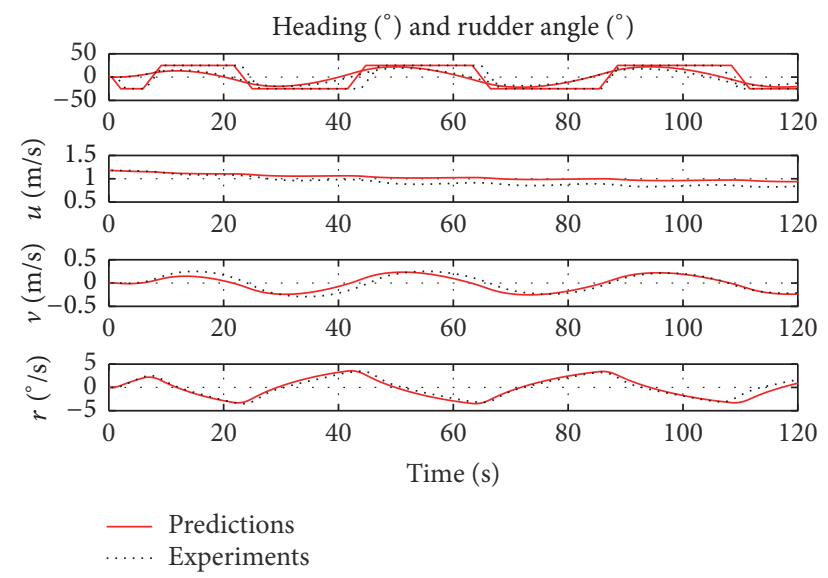

Figure 2: Prediction of $25^{\circ} / 5^{\circ}$ zigzag manoeuvre of a KCLCC2 ship.
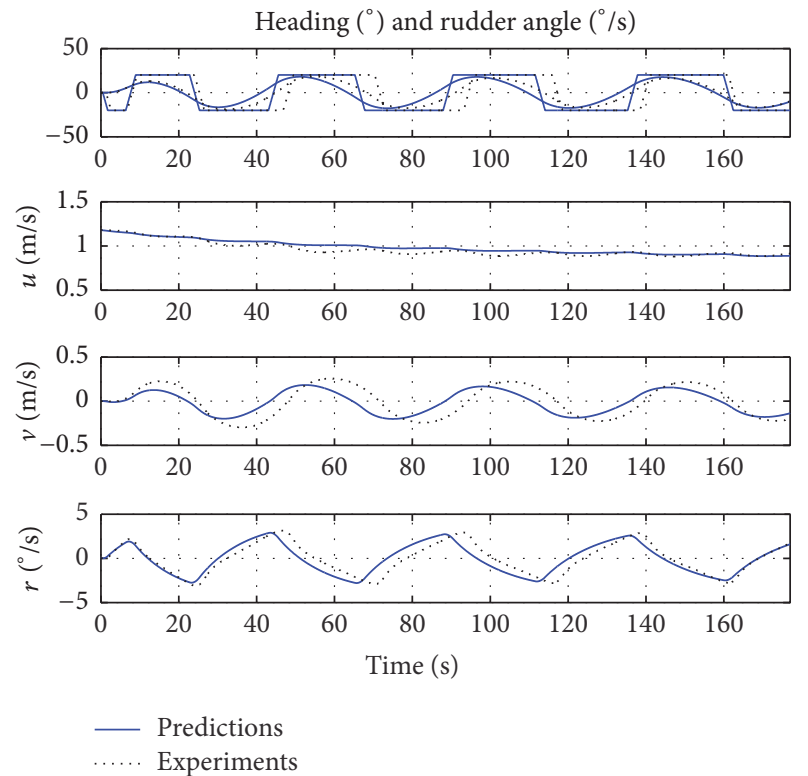

FIGURE 3: Prediction of $20^{\circ} / 5^{\circ}$ zigzag manoeuvre of a KCLCC2 ship.

experiment is achieved. It is owing not only to the use of SVM method, a powerful modeling tool, but also to the measures taken to diminish the parameter drift.

\section{Conclusions}

Parameter identifiability is an important issue for the SI application to the modeling of ship manoeuvring. Some parameters in the manoeuvring model cannot be obtained using SI, while some parameters deviate from true values even if they can be derived. Several measures are proposed to deal with the problem of parameter identifiability and the validities are demonstrated by examples. To determine the added masses and added moment of inertia, slender-body theory provides a simple estimation method. To diminish the parameter drift effect, the mathematical model of ship manoeuvring motion is simplified on the basis of regression analysis;
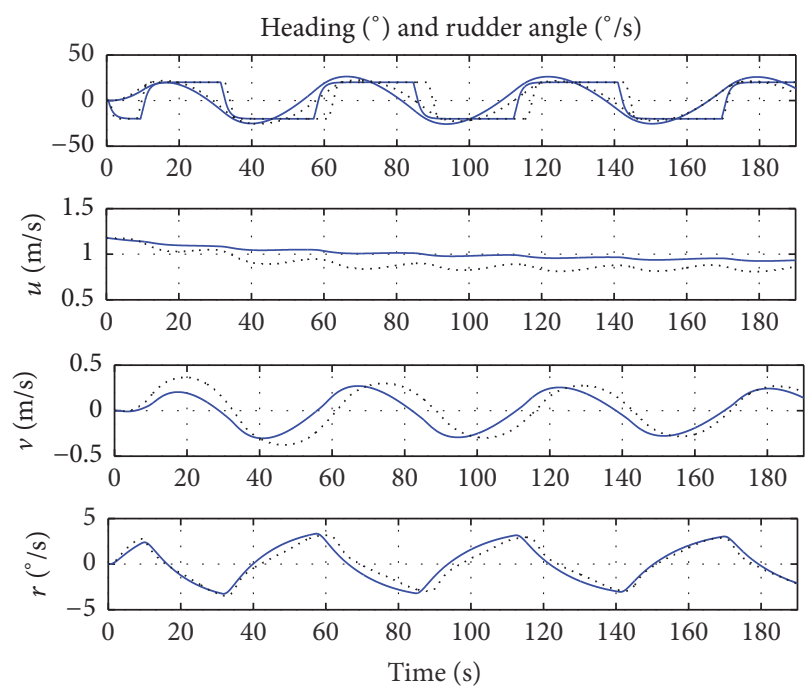

- Predictions

Experiments

FIgURE 4: Prediction of $20^{\circ} / 10^{\circ}$ zigzag manoeuvre of a KCLCC2 ship.

difference of the samples and additional signal also take effect. These measures can be viewed as developed approaches that are commonly used in regression analysis to diminish the multicollinearity. For instance, the simplification of a model can be viewed as a kind of pruning-variable method or PCR analysis since the main components in the model remained while some trivial variables are removed. The additional signal method can be viewed as the opposition to pruningvariable method.

Future work will be devoted to the study on a more accurate, practical and universal estimation method for added masses and added moment of inertia since slender-body method gives satisfactory results only for slender ships and "mild" manoeuvres. Moreover, how to design an appropriate additional signal according to the characteristics of samples will be studied.

\section{Competing Interests}

The author declares that they have no competing interests.

\section{Acknowledgments}

This work was partially supported by the Special Item for University in Fujian Province supported by the Education Department (no. JK15003) and the Special Item supported by the Fujian Provincial Department of Ocean and Fisheries (no. MHGX-16). The author is grateful to the Hamburg Ship Model Basin (HSVA) for providing the experimental data.

\section{References}

[1] International Maritime Organization (IMO), "Standards for ship manoeuvrability," Resolution MSC 137(76), 2002. 
[2] The Maneuvering Committee of ITTC, "Final report and recommendations to the 25th ITTC", in Proceedings of the 25th International Towing Tank Conference, pp. 143-208, Fukuoka, Japan, 2008.

[3] G. Rajesh and S. K. Bhattacharyya, "System identification for nonlinear maneuvering of large tankers using artificial neural network," Applied Ocean Research, vol. 30, no. 4, pp. 256-263, 2008.

[4] L. Moreira and C. Guedes Soares, "Recursive neural network model of catamaran manoeuvring," International Journal of Maritime Engineering, vol. 154, pp. A121-A130, 2012.

[5] W. L. Luo and Z. J. Zou, "Blind prediction of ship manoeuvring by using Support Vector Machines," in Proceedings of the the 29th International Conference on Offshore Mechanics and Arctic Engineering (OMAE '10), Shanghai, China, June 2010.

[6] W. Luo, L. Moreira, and C. Guedes Soares, "Manoeuvring simulation of catamaran by using implicit models based on support vector machines," Ocean Engineering, vol. 82, pp. 150159, 2014.

[7] T. Holzhüter, "Robust identification in an adaptive track controller for ships," in Proceedings of the 3rd IFAC Symposium on Adaptive Systems in Control and Signal Processing, pp. 275-280, Glasgow, UK, 1989.

[8] R. Muñoz-Mansilla, J. Aranda, J. M. Díaz, and J. de la Cruz, "Parametric model identification of high-speed craft dynamics," Ocean Engineering, vol. 36, no. 12-13, pp. 1025-1038, 2009.

[9] M. N. Hayes, Parameters identification of nonlinear stochastic systems applied to ocean vehicle dynamics [Ph.D. thesis], Massachusetts Institute of Technology, Cambridge, Mass, USA, 1971.

[10] J. Van Amerongen, "Adaptive steering of ships-a model reference approach," Automatica, vol. 20, no. 1, pp. 3-14, 1984.

[11] M. A. Abkowitz, "Measurement of hydrodynamic characteristic from ship maneuvering trials by system identification," Transactions of Society of Naval Architects and Marine Engineers, vol. 88, pp. 283-318, 1981.

[12] L. P. Perera, P. Oliveira, and C. Guedes Soares, "System identification of nonlinear vessel steering," Journal of Offshore Mechanics and Arctic Engineering, vol. 137, no. 3, Article ID 031302, pp. 1-7, 2015.

[13] K. J. Åström, "Maximum likelihood and prediction error methods," Automatica, vol. 16, no. 5, pp. 551-574, 1980.

[14] C. G. Källström and K. J. Åström, "Experiences of system identification applied to ship steering," Automatica, vol. 17, no. 1, pp. 187-198, 1981.

[15] W.-W. Zhou and M. Blanke, "Identification of a class of nonlinear state-space models using RPE techniques," IEEE Transactions on Automatic Control, vol. 34, no. 3, pp. 312-316, 1989.

[16] E. Revestido Herrero and F. J. Velasco González, “Two-step identification of non-linear manoeuvring models of marine vessels," Ocean Engineering, vol. 53, pp. 72-82, 2012.

[17] R. P. Selvam, S. K. Bhattacharyya, and M. Haddara, "A frequency domain system identification method for linear ship maneuvering," International Shipbuilding Progress, vol. 52, no. 1, pp. 5-27, 2005.

[18] S. K. Bhattacharyya and M. R. Haddara, "Parametric identification for nonlinear ship maneuvering," Journal of Ship Research, vol. 50, no. 3, pp. 197-207, 2006.

[19] Y. Chen, Y. Song, and M. Chen, "Parameters identification for ship motion model based on particle swarm optimization," Kybernetes, vol. 39, no. 6, pp. 871-880, 2010.
[20] Y. T. Dai, L. Q. Liu, and S. S. Feng, "On the identification of coupled pitch and heave motions using opposition-based particle swarm optimization," Mathematical Problems in Engineering, vol. 2014, Article ID 784049, 10 pages, 2014.

[21] K.-P. Rhee, S.-Y. Lee, and Y.-J. Sung, "Estimation of manoeuvring coefficients from PMM test by genetic algorithm," in Proceedings of the International Symposium and Workshop on Force Acting on a Manoeuvring Vessel, Val de Reuil, France, 1998.

[22] S. Sutulo and C. Guedes Soares, "An algorithm for offline identification of ship manoeuvring mathematical models from free-running tests," Ocean Engineering, vol. 79, pp. 10-25, 2014.

[23] W. L. Luo and Z. J. Zou, "Parametric identification of ship maneuvering models by using support vector machines," Journal of Ship Research, vol. 53, no. 1, pp. 19-30, 2009.

[24] W. L. Luo, C. Guedes Soares, and Z. J. Zou, "Parameter identification of ship maneuvering model based on support vector machines and particle swarm optimization," Journal of Offshore Mechanics and Arctic Engineering, vol. 138, no. 3, Article ID 031101, 8 pages, 2016.

[25] W. Y. Hwang, "Cancellation effect and parameter identifiability of ship steering dynamics," International Shipbuilding Progress, vol. 29, no. 332, pp. 90-120, 1982.

[26] C. G. Källström, "Identification and adaptive control applied to ship steering," Tech. Rep. SSPA/PUB-93, 1982.

[27] H. D. Nguyen, "Recursive identification of ship maneuvering dynamics and hydrodynamics," in Proceedings of the 36th EMAC Annual Conference, pp. C717-C732, Reykjavik, Iceland, 2007.

[28] F. J. Velasco, I. Zamanillo, E. Lopez, and E. Moyano, "Parameter estimation of ship linear maneuvering models," in Proceedings of the OCEANS 2011 IEEE Santander Conference, pp. 1-8, Santander, Spain, June 2011.

[29] M. R. Haddara and Y. Wang, "Parametric identification of manoeuvring models for ships," International Shipbuilding Progress, vol. 46, no. 445, pp. 5-27, 1999.

[30] D. Hess, W. Faller, J. Lee, T. Fu, and E. Ammeen, "Ship maneuvering simulation in wind and waves: a nonlinear time-domain approach using recursive neural networks," in Proceedings of the 26th Symposium on Naval Hydrodynamics, Rome, Italy, 2006.

[31] D. Hess, W. Faller, and J. Lee, "Real-time nonlinear simulation of maneuvers for U.S. navy combatant DTMB 5415," in Proceedings of the Workshop on Verification and Validation of Ship Maneuvering Simulation Methods (SIMMAN '08), pp. E15-E21, Copenhagen, Denmark, 2008.

[32] M. A. Abkowitz, "Lectures on ship hydrodynamics—steering and manoeuvrability," Tech. Rep. Hy-5, Hydro- and Aerodynamics Laboratory, Lyngby, Denmark, 1964.

[33] A. Ogawa and H. Kasai, "On the mathematical model of manoeuvring motion of ships," International Shipbuilding Progress, vol. 25, no. 292, pp. 306-319, 1978.

[34] K. Nomoto, T. Tagushi, K. Honda, and S. Hirano, "On steering qualities of ships," International Shipbuilding Progress, vol. 4, no. 35, pp. 354-370, 1957.

[35] S. Toxopeus, "Validation of slender-body method for prediction of linear manoeuvring coefficients using experiments and viscous-flow calculations," in Proceedings of the 7th ICHD International Conference on Hydrodynamics (ICHD '06), pp. 589-598, University of Naples "Federico II", October 2006.

[36] T.-I. Lee, K.-S. Ahn, H.-S. Lee, and D.-K. Yum, "On an empirical prediction of hydrodynamic coefficients for modern ship hulls," in Proceedings of the International Conference on 
Marine Simulation and Ship Manoeuvrability (MARSIM '03), Kanazawa, Japan, August 2003.

[37] H. Norrbin Nils, "Theory and observations on the use of a mathematical model for ship manoeuvring in deep and confined waters," Report No. SSPA-Pub-68, Swedish State Shipbuilding Experimental Tank Goteborg, 1971.

[38] R.-C. Zhu, H.-Q. Guo, G.-P. Miao, and J.-W. Yu, “Computational method for evaluation of added mass and damping of ship based on CFD theory," Journal of Shanghai Jiaotong University, vol. 43, no. 2, pp. 198-203, 2009.

[39] J. N. Newman, Marine Hydrodynamics, MIT Press, Cambridge, Mass, USA, 1977.

[40] M. S. Chislett and J. Strom-Tejsen, "Planar motion mechanism tests and full-scale steering and maneuvering predictions for a Mariner class vessel," International Shipbuilding Progress, vol. 12, pp. 201-224, 1965.

[41] M. Z. Fabrycy, "Multicollinearity caused by specification errors," Applied Statistics, vol. 24, no. 2, pp. 250-254, 1975.

[42] W. L. Luo, On the modeling of ship manoeuvring by using support vector machines [Ph.D. thesis], Shanghai Jiao Tong University, Shanghai, China, 2009.

[43] S. S. Bowles and H. M. Levin, "More on multicollinearity and the effectiveness of schools," The Journal of Human Resources, vol. 3, no. 3, pp. 393-400, 1968.

[44] I. Jolliffe, Principal Component Analysis, John Wiley \& Sons, 2002.

[45] S. Wold, A. Ruhe, H. Wold, and W. J. Dunn III, “The collinearity problem in linear regression. The Partial Least Squares (PLS) approach to generalized inverses," SIAM Journal on Scientific and Statistical Computing, vol. 5, no. 3, pp. 735-743, 1984.

[46] A. G. Ivakhnenko and J. A. Muller, "Present state and new problems of further GMDH development," Systems Analysis Modeling Simulation, vol. 20, pp. 3-16, 1995.

[47] H. K. Yoon and K. P. Rhee, "Identification of hydrodynamic coefficients in ship maneuvering equations of motion by Estimation-Before-Modeling technique," Ocean Engineering, vol. 30, no. 18, pp. 2379-2404, 2003.

[48] S. M. Yeon, D. J. Yeo, and K. P. Rhee, "Optimal input design for the identification of low-speed manoeuvring mathematical model," in Proceedings of the International Conference on Marine Simulation and Ship Manoeuvrability (MARSIM '06), Terschelling, The Netherlands, June 2006.

[49] W. Y. Hwang, Application of system identification to ship maneuvering [Ph.D. thesis], Massachusetts Institute of Technology, Cambridge, Mass, USA, 1980.

[50] K. P. Rhee and K. Kim, "A new sea trial method for estimating hydrodynamic derivatives," Ship Ocean Technology, vol. 3, no. 3, pp. 25-44, 1999.

[51] M. Viviani, R. Depascale, L. Sebastiani, C. Podenzana Bonvino, R. Dattola, and M. Soave, "Alternative methods for the identification of hydrodynamic derivatives from standard manoeuvres," in Proceedings of the International Conference on Marine Simulation and Ship Manoeuvrability (MARSIM '03), Kanazawa, Japan, August 2003.

[52] W.-L. Luo and Z.-J. Zou, "Elimination of simultaneous drift and sensitivity analysis in the hydrodynamic modeling of ship manoeuvring," Journal of Shanghai Jiaotong University, vol. 42, no. 8, pp. 1358-1362, 2008.

[53] R. R. Shenoi, P. Krishnankutty, and R. P. Selvam, "Sensitivity study of hydrodynamic derivative variations on the maneuverability prediction of a container ship," in Proceedings of the
ASME 34th International Conference on Ocean, Offshore and Arctic Engineering (OMAE '15), American Society of Mechanical Engineers, June 2015.

[54] W. L. Luo and Z. J. Zou, "Analysis of captive model oblique towing test by using least squares support vector machines," Shipbuilding of China, vol. 51, no. 2, pp. 10-14, 2010. 


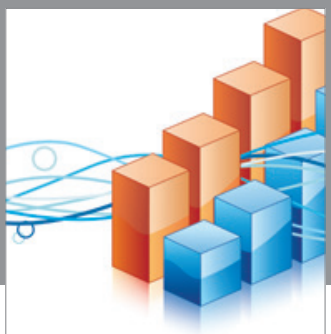

Advances in

Operations Research

vatem alat4

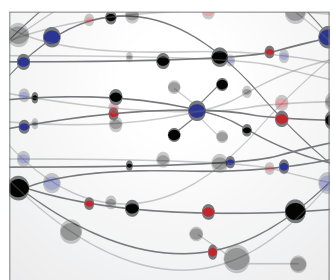

\section{The Scientific} World Journal
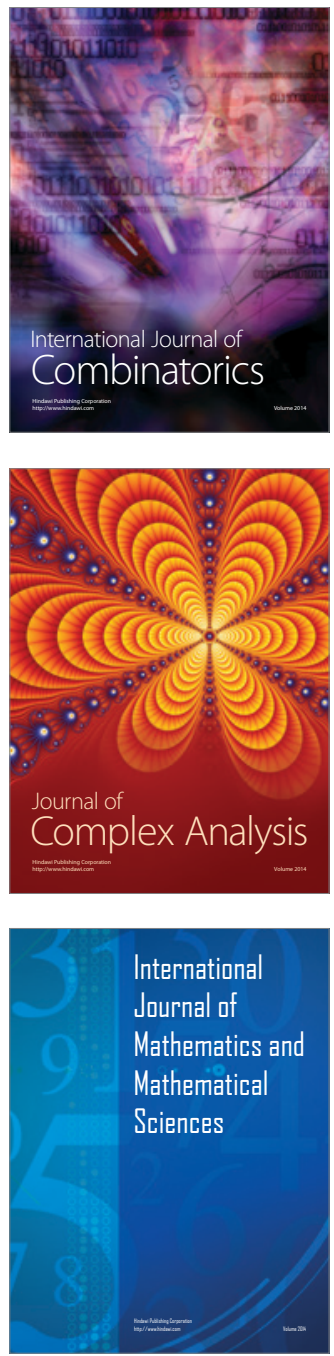
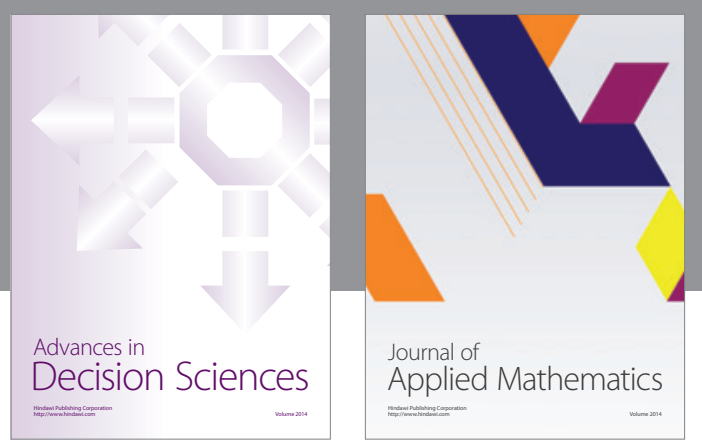

Algebra

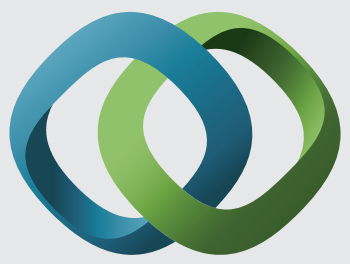

\section{Hindawi}

Submit your manuscripts at

http://www.hindawi.com
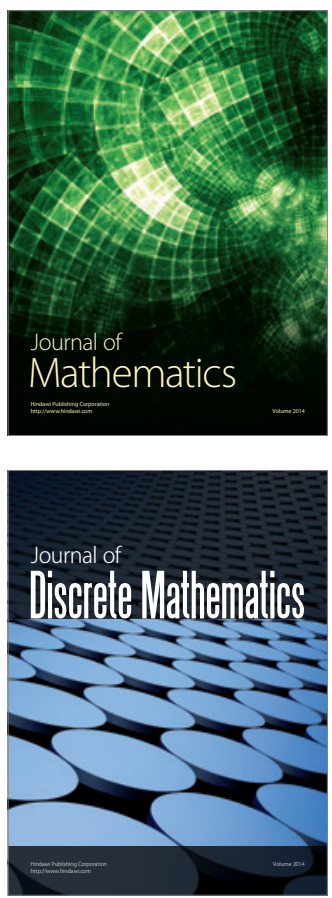

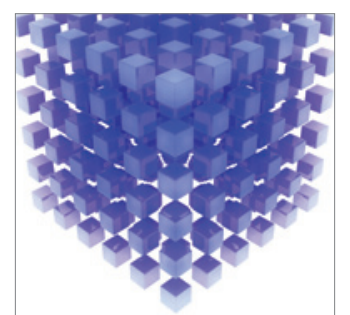

Mathematical Problems in Engineering
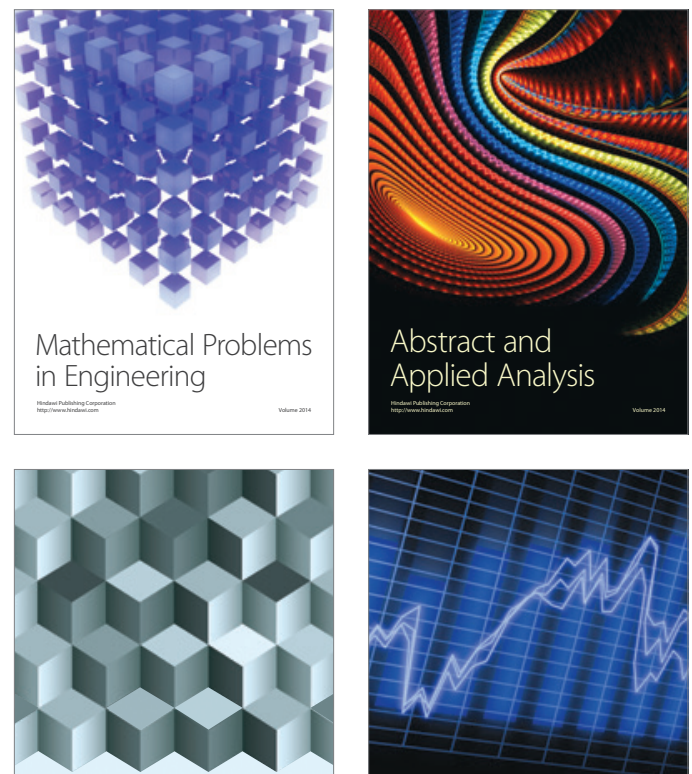

Journal of

Function Spaces

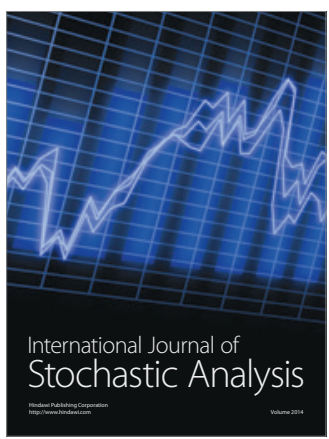

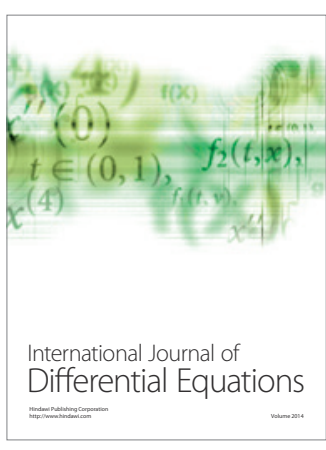
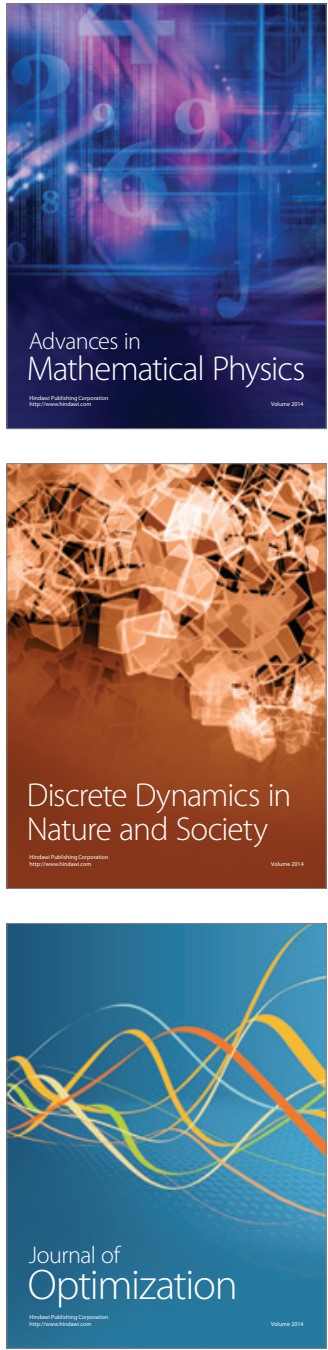